\section{Nanoparticles for bioanalysis}

\author{
María Carmen Blanco-López ${ }^{1}$ (D) $\cdot$ Montserrat Rivas $^{2}$
}

Published online: 4 March 2019

(C) Springer-Verlag GmbH Germany, part of Springer Nature 2019

The materials available at every period of history determine the civilizations' quality of life. From the Stone Age, Copper Age, Bronze Age and Iron Age to ceramics, steel, plastics, and semiconductors, we have now reached the nanomaterials age. Nanoparticles (NPs), with sizes in the 1- to 100-nm range, exhibit properties that differ markedly from those of the bulk. The study of such properties is triggering novel solutions for our needs in health and energy.

In the field of analytical chemistry, they offer new tools to overcome the limitations of current methods and to develop innovative devices. This is due to their unique features related very often to compatibility with biomolecules and advantageous magnetic, optical, or catalytic properties. The great challenge is to lower limits of detection, by providing signal amplification, together with simplification of the protocols. The reviews and research papers in this topical collection give an insight into the current achievements and challenges in analytical-based devices for biomedical and food science fields. They represent leading directions in electroanalysis, spectroscopy, and chromatographic portable platforms. Particular attention is paid to emerging innovative transduction systems with magnetic nanoparticles.

In electroanalysis, nanostructures have been used as electrode modifiers for the immobilization of bioreceptors, as signal tag systems replacing enzymes at immunosensors or genosensors, catalysts of electron transfer processes, or a combination of these features. From metallic (Au, Pt) NPs to carbon nanomaterials (graphene, carbon NPs, and nanotube scaf-

Published in the topical Collection Nanoparticles for Bioanalysis with guest editors María Carmen Blanco-López and Montserrat Rivas.

María Carmen Blanco-López

cblanco@uniovi.es

1 Department of Physical and Analytical Chemistry, University of Oviedo, 33006 Oviedo, Spain

2 Department of Physics, Gijón Polytechnic School, University of Oviedo, 33204 Gijón, Spain folds), NPs provide a large surface area and high conductivity, catalytic behavior, and compatibility to retain bioactivity. This is especially relevant to overcome challenges related to the determination of nucleic acids and miRNAs in particular, where packing determines hybridization efficiency and a good control of orientation is required for reproducibility. Semiconductor quantum dots (QDs) or metal-organic framework nanoparticles (clusters of metal ions linked by organic bridging ligands) are replacing enzymes in their traditional roles as electroactive tags and catalytic centers. This results in more robust devices, with higher thermal and chemical stability, shorter analysis times, and lower cost. All these advantages add up to the easy miniaturization inherent to electrochemical devices, enabling portable instrumentation for point-of-care clinical demand. However, electrochemical nucleic acid biosensors are still restricted to research laboratories. The simultaneous detection of multiple targets in complex samples needs to be solved for translation of these devices into real-world settings.

This is also a goal for spectroscopy-based nanosensors. miRNAs have great potential as diagnostic or disease progression biomarkers, but the technical and instrumental requirements of current analytical platforms based on quantitative polymerase chain reaction (PCR) or sequencing microarrays limit their spread for clinical use and biomedical research. The collection of data in large patient cohorts for screening or research purposes demands simplification of the protocols. The large surface area-to-volume ratio of AuNPs and interface-dominated properties are also interesting for innovative direct multianalyte detection of miRNAs without enhancing protocols, and new possibilities are open for microarray imaging techniques.

Cutting-edge research in spectroscopy targets also new means of detection. For example, by coating an attenuated total reflection (ATR) waveguide with carbon dots, IR signal enhancement effects were found for aromatic dyes and purine derivatives. This effect was used at a surface-enhanced infrared absorption (SEIRA) method for adenine determination, and encourages the use of SEIRA nanostructures for the 
development of label-free IR-based bioassays. Carbon dots exhibit advantages over semiconductor QDs, such as biocompatibility, easy and sustainable production routes, chemical stability, and easy dispersion in aqueous media.

Immunochromatographic tests and lateral flow strip biosensors are very favorable to meet the point-of-care requirements and market translation. Many AuNP-based paper in vitro tests have already reached society due to their simplicity, specificity, robustness, short analysis times, cost-effectiveness, and portability. AuNPs provide acceptable sensitivity for many applications (at the nanomolar level), due to the high extinction coefficient that results from localized surface plasmon resonance enhancement of the electromagnetic field in the visible range of the spectrum. Multiplexed determinations can be developed by combining different metallic NPs (AuNPs, AgNPs) for detection at different test lines. This has been successfully applied for the determination of food allergens. However, the bottleneck for the wide use of these platforms in a clinical setting is still the need to achieve high sensitivity in complex biological samples, with minimum sample treatment.

Magnetic nanoparticles have been traditionally used for selective capture and separation of a target analyte from the sample matrix. However, their potential as a transducer for the development of new analytical instruments has not yet been fully explored. Control of the synthesis of magnetic nanoparticles, standardization of protocols to estimate their iron content, and their correlation with fundamental studies are important in order to rationally develop and optimize novel strategies for sensing. Flow cytometry and biosensors for biomedical or food safety monitoring are among this family of emerging devices. Instruments based on magnetic properties have a great potential to meet the highly demanding needs for both signal amplification and effective isolation, with low sample volume requirements. It is expected that they will also provide solutions for the quantification and isolation of extracellular vesicles (exosomes), which in recent years have attracted attention as a new source of biomarkers.

Further improvements in the sensing field could result by synergies among different nanoparticles and transducers. We hope that the selection of papers in this topical collection encourages the search for innovative nanoparticle-based strategies for a novel generation of biosensors.

We would like to thank all authors for their valuable contributions to this issue, and the NanoBioAp network that has inspired this topical collection. We also acknowledge the constructive comments of referees, aiming to keep high-quality standards. Finally, we would like to thank Professor Alfredo
Sanz Medel and the Editorial Office and Editors for their support and assistance during the preparation of this issue.

Publisher's note Springer Nature remains neutral with regard to jurisdictional claims in published maps and institutional affiliations.

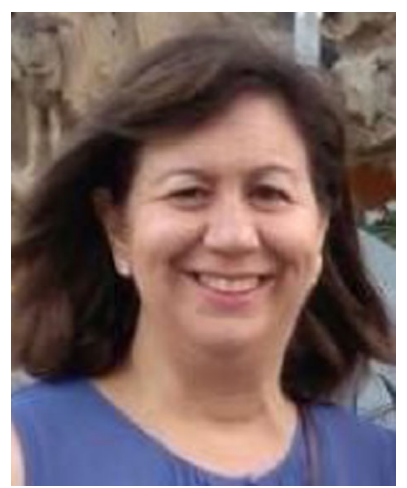

María Carmen Blanco-López is Associate Professor at the Department of Physical and Analytical Chemistry, at the University of Oviedo, Spain. She got her PhD at the University of Leeds (UK, 1997). She has worked with materials characterization and the design of synthetic receptors based on molecularly imprinted polymers. She has developed electrochemical sensors for the detection of biomarkers, pharmaceuticals, and pollutants. Her current research involves in vitro rapid tests based on lateral flow immunoassays and the development of amplification strategies based on nanomaterials. These include metallic and magnetic nanoparticles, and nanovesicles (liposomes, niosomes). These materials could also be applied to drug release and extracellular vesicle (exosome) biomimetic systems. She has developed biosensors for biomarkers (pneumolysin, PSA, troponin), exosomes, and allergenics (histamine) by coupling these immunochromatographic tests to optical, electrochemical, and magnetic readers. She coordinates the H2020-MSC Innovative Training Network "Break Biofilms", and with Montserrat Rivas, the national Cluster of Excellence "Nanoparticles for Bioanalysis" (NanoBioAp, www.nanobioap.org).

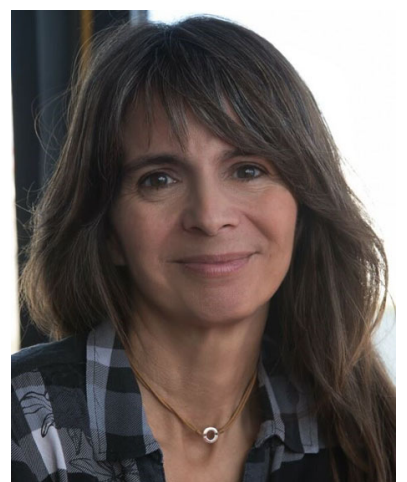

Montserrat Rivas currently works at the Department of Physics, University of Oviedo. She has a degree and a PhD in physics, and during her studies she carried out research in Laboratoire de Magnetisme et Optique (CNRS) de Bellevue (Paris) and Laboratoire Louis Néel (CNRS) de Grenoble (France). Nowadays, she researches in magnetic materials with a special focus on bioapplications of magnetic nanoparticles and bio-sensing. She coleads a multidisciplinary group specialized in magnetic lateral flow immunoassays for point-of-care bioanalysis. Her main project is "Superparamagnetic nanoparticle-based biosensor for non-invasive early diagnosis of colorectal cancer." 